Pacific Journal of 


\title{
ON THE LATTICE OF VARIETIES OF BANDS OF GROUPS
}

\author{
T. E. HALL AND P. R. JONES
}

In this paper we prove that the lattice of varieties of bands of groups is modular and apply this to direct decompositions of various sublattices. The join of the varieties of bands and of completely simple semigroup is shown to be the variety of "pseudo-orthodox" bands of groups.

1. Introduction. When considered as semigroups with an additional unary operation $x \rightarrow x^{-1}$, where $x^{-1}$ denotes the (unique) inverse of $x$ in the subgroup to which it belongs, the class $\boldsymbol{C R}$ of completely regular semigroups (often called unions of groups) forms a variety of universal algebras, containing as a subvariety thevariety $\boldsymbol{B} \boldsymbol{G}$ of bands of groups (those completely regular semigroups on which $\mathscr{H}$ is a congruence) ([12]). In this paper results of Spitznagel [14] on the lattice of congruences on a band of groups are applied to show that $\mathscr{V}(\boldsymbol{B} \boldsymbol{G})$, the lattice of subvarieties of $\boldsymbol{B G}$, is modular (Theorem 3.1). Petrich $[12,13]$ considered various subvarieties of $\boldsymbol{B} \boldsymbol{G}$ but left open the problem [13, p.1196] of finding the join of the subvarieties $\boldsymbol{B}$ and CS (of bands and of completely simple semigroups respectively). We show that $\boldsymbol{B} \vee \boldsymbol{C S}=\boldsymbol{P O B G}$, the variety of pseudo-orthodox bands of groups, and is thus strictly contained in $\boldsymbol{B} \boldsymbol{G}$. (If $\boldsymbol{V}$ is a variety of completely regular semigroups and $S \in \boldsymbol{C R}$ we shall call $S$ pseudo- $\boldsymbol{V}$ if $e S e \in V$ for every idempotent $e$ of $S$.) This result is actually an immediate corollary to our characterization of the join $\boldsymbol{O} \vee \boldsymbol{N B G}$ of the varieties of orthodox completely regular semigroups and of normal bands of groups. Theorem 3.1 is also applied to directly decompose various sublattices of $\mathscr{V}(\boldsymbol{B} \boldsymbol{G})$.

2. Preliminaries. For background to this paper the reader is referred to [13] where defining identities are presented for most of the varieties encountered here. Various subvarieties of $\boldsymbol{C R}$ are shown on the diagram on p. 1172 of [13]. For easy reference we will give a list of our abbreviations for these:

$\boldsymbol{C R}$ : completely regular semigroups

$B G$ : bands of groups

$N B G$ : normal bands of groups

$O B G$ : orthodox bands of groups

$B$ : bands

CS: completely simple semigroups

NB: normal bands 
$S L G:$ semilattices of groups

$\boldsymbol{R} G$ : rectangular groups

$S L:$ semilattices

$\boldsymbol{R B}$ : rectangular bands

$G$ : groups

$T:$ the trivial variety

Further $\boldsymbol{O}$ will denote the variety of orthodox completely regular semigroups (those whose set of idempotents forms a subsemigroup).

We will otherwise use the terminology and notation of [7] (for semigroup theory) and [6] (for lattice theory). Throughout $E_{S}$ denotes the set of idempotents of the semigroup $S$ and $\left\langle E_{S}\right\rangle$ the subsemigroup of $S$ generated by $E_{S}$ (completely regular, by [3], when $\boldsymbol{S} \in \boldsymbol{C R})$.

If $\rho$ is a relation on $S$ then $\rho^{\sharp}$ will denote the congruence on $S$ generated by $\rho$ and if $A \subseteq S, \rho \mid A$ will denote the restriction $\rho \cap$ $(A \times A)$ of $\rho$ to $A$. The symbol $\Lambda(S)$ represents the lattice of congruences on $S$, with $\iota$ and $\omega$ the smallest and largest elements respectively. A point which will be of importance in $\S 3$, in particular, is that $\Lambda(S)$ is a sublattice of the lattice $\Sigma(S)$ of all equivalences on $S$ ([7], §I. 5).

3. Bands of groups. We will prove the following theorem.

Theorem 3.1. The lattice $\mathscr{V}(\boldsymbol{B} G)$ is modular.

Before beginning the proof we need some lattice-theoretic concepts from [6], §III. 2. If $L$ is a lattice and $a \in L$ then $a$ is said to be neutral if

(i ) a "separates" $L$ :

if $x, y \in L$ then $a \wedge x=a \wedge y$ and $a \vee x=a \vee y$ together imply $x=y$,

and

(ii) the map $x \rightarrow a \vee x$ is a morphism

(iii) the map $x \rightarrow a \wedge x$ is a morphism.

Elements satisfying (i) and (ii) are called standard. It is almost immediate that $a$ is neutral if and only if the map $x \rightarrow(a \wedge x, a \vee x)$ is an isomorphism of $L$ upon a subdirect product of the sublattices $\{x \in L: x \leqq a\}$ and $\{x \in L: x \geqq a\}$. Clearly neutrality is a self-dual notion. (For equivalent formulations of neutrality see [6], Theorem III. 2.4.)

We now quote some results of Spitznagel, rephrased in the above terminology.

RESUlt 3.2 [14, Theorem 3.9]. On any band of groups $S$, the 
congruence $\mathscr{H}$ is a standard element of $\Lambda(S)$. Further it is a neutral element of the sublattice $[\iota, \mathscr{D}]$ of $\Lambda(S)$.

REMARK 3.3. Using similar methods to those of Spitznagel it may be verified that an analogous result is true in any completely regular semigroup $S$. For example $\mathscr{H}$ separates $\Lambda(S)$ (in the above sense), the map $\rho \rightarrow \rho \vee \mathscr{H}$ is a complete morphism of the sublattice $\Lambda(S)$ of $\Sigma(S)$ (see $\S 2$ ) into $\Sigma(S)$ and the map $\rho \rightarrow \rho \cap \mathscr{H}$ is a complete morphism of the sublattice $[\iota, \mathscr{D}]$ of $\Lambda(S)$ into $\Sigma(S)$ (the join being interpreted as join of equivalences where necessary). A consequence of the last statement will be used in the proof of Theorem 5.3: for any collection $\left\{\tau_{k}\right\}_{k \in K}$ of congruences on $S$, each contained in $\mathscr{D}, \vee\left\{\tau_{k}: k \in K\right\} \cap \mathscr{C}=V\left\{\tau_{k} \cap \mathscr{H}: k \in K\right\}$, so that in particular, if $\tau_{k} \cap \mathscr{H}=\imath$ for each $k \in K$ then $V\left\{\tau_{k}: k \in K\right\} \cap \mathscr{H}=\iota$.

Now in any variety of algebras the lattice of subvarieties is dually isomorphic with the lattice of fully invariant congruences on the free algebra $F$ on a countable set of generators in that variety, and moreover the lattice of fully invariant congruences on any algebra $A$ forms a sublattice of the lattice of all congruences on $A$. (See, for example [10]. A congruence $\rho$ on an algebra $A$ is fully invariant if $a \rho b$ implies $a \theta \rho b \theta$ for every endomorphism $\theta$ of $A$.)

Noticing that both $\mathscr{H}$ and $\mathscr{D}$ are fully invariant congruences on any band of groups, we see from Result 3.2 that in this case $\mathscr{H}$ is a neutral element in the lattice of fully invariant congruences contained in $\mathscr{D}$. Since $\mathscr{H}$ defines the variety $\boldsymbol{B}$ (within $\boldsymbol{B} \boldsymbol{G}$ ) and $\mathscr{D}$ defines $\boldsymbol{S L}$, we see that $\boldsymbol{B}$ is a neutral element in the sublattice $[\boldsymbol{S L}, \boldsymbol{B} \boldsymbol{G}]$ of $\mathscr{V}(\boldsymbol{B} \boldsymbol{G})$. We then have

Proposition 3.4. The sublattice $[\boldsymbol{S L}, \boldsymbol{B G}]$ is a subdirect product of the sublattices $[\boldsymbol{S L}, \boldsymbol{B}]$ and $[\boldsymbol{B}, \boldsymbol{B G}]$ and is therefore modular.

Proof. The first statement follows from the remarks on the definition of neutrality. For the second, we quote the result of $[1,2,4]$ that $\mathscr{V}(\boldsymbol{B})$ is distributive (whence modular) and then note again that since $\boldsymbol{B}$ corresponds to the fully invariant congruence $\mathscr{H}$ on $F$ (the free band of groups on a countable set of generators), the sublattice $[\boldsymbol{B}, \boldsymbol{B} \boldsymbol{G}]$ is dually isomorphic with the lattice of all fully invariant congruences on $F$ contained in $\mathscr{H}$, which in turn is a sublattice of the lattice of all congruences on $F$ contained in $\mathscr{H}$. Since this last lattice is modular [9], so is $[\boldsymbol{B}, \boldsymbol{B G}]$ and therefore $[S L, B G]$ also.

The proof of Theorem 3.1 will be completed by the following 
proposition, since $\mathscr{V}(S L)$ consists of just $\boldsymbol{T}$ and $S L$ and is therefore distributive. Note that this result is true for arbitrary varieties containing $\boldsymbol{S L}$ (and contained in $\boldsymbol{C R}$ ).

Proposition 3.5. The variety $\boldsymbol{S L}$ is a neutral element in $\mathscr{C}(\boldsymbol{C R})$ and therefore in any sublattice containing $\boldsymbol{S L}$. In particular, therefore, $\mathscr{V}(\boldsymbol{B} \boldsymbol{G})$ is a subdirect product of $\mathscr{V}(\boldsymbol{S L})$ and $[\boldsymbol{S L}, \boldsymbol{B G}]$.

Proof. It will prove convenient here to show directly that the map $\chi: K \rightarrow(K \cap S L, K \vee S L)$ is an isomorphism of $\mathscr{V}(\boldsymbol{C R})$ into $\mathscr{V}(\boldsymbol{S L}) \times[\boldsymbol{S L}, \boldsymbol{C R}]$. Clearly $\chi$ is order-preserving.

Suppose, on the other hand, that $\boldsymbol{K}, \boldsymbol{L} \subseteq \boldsymbol{C R}$ and $\boldsymbol{K} \cap \boldsymbol{S L} \subseteq \boldsymbol{L} \cap$ $\boldsymbol{S} \boldsymbol{L}, \boldsymbol{K} \vee \boldsymbol{S L} \subseteq \boldsymbol{L} \vee \boldsymbol{S L}$. Now since $\mathscr{V}(\boldsymbol{S L})=\{\boldsymbol{T}, \boldsymbol{S L}\}$, either $\boldsymbol{S L} \subseteq \boldsymbol{L}$ or $\boldsymbol{L} \cap \boldsymbol{S} \boldsymbol{L}=\boldsymbol{T}$. In the former case $\boldsymbol{K} \leqq \boldsymbol{K} \vee \boldsymbol{S} \boldsymbol{L} \subseteq \boldsymbol{L} \vee \boldsymbol{S L}=\boldsymbol{L}$. Otherwise $\boldsymbol{L} \cap \boldsymbol{S L}=\boldsymbol{T}$, whence $\boldsymbol{K} \cap \boldsymbol{S L}=\boldsymbol{T}$ also. But on any completely regular semigroup $S, \mathscr{D}$ is an $S L$-congruence and if $S / \mathscr{D} \in \boldsymbol{T}$ then $S \in C S$. Hence $K, L \subseteq C S$, and so $K \vee S L, L \vee S L \subseteq C S \vee S L=$ $N B G$.

From [13, Theorem 4.7], the map $\boldsymbol{V} \rightarrow \boldsymbol{V} \cap \boldsymbol{C S}$ is a lattice morphism of $\mathscr{C}(\boldsymbol{N B G})$ upon $\mathscr{V}(\boldsymbol{C S})$. Thus $\boldsymbol{K}=(\boldsymbol{K} \cap \boldsymbol{C S}) \vee(\boldsymbol{S L} \cap \boldsymbol{C S})=$ $(\boldsymbol{K} \vee \boldsymbol{S L}) \cap \boldsymbol{C S}$ and similarly $\boldsymbol{L}=(\boldsymbol{L} \vee \boldsymbol{S L}) \cap \boldsymbol{C S}$, whence $\boldsymbol{K} \vee \boldsymbol{S} \boldsymbol{L} \cong$ $\boldsymbol{L} \vee \boldsymbol{S} \boldsymbol{L}$ implies $\boldsymbol{K} \cong \boldsymbol{L}$.

Hence $\chi$ is an order isomorphism and $S \boldsymbol{L}$ is neutral.

CoROLlaRy 3.6. The lattice $\mathscr{Y}(C S)$ is modular.

From Theorem 3.1, direct decompositions of various sublattices of $\mathscr{V}(\boldsymbol{B} \boldsymbol{G})$ may be obtained by applying the following result.

RESULT 3.7 ([6], Theorem IV. 1.14). If $a, b$ are elements of a modular lattice $L$ then the sublattice of $L$ generated by $[a \wedge b, a]$ and $[a \wedge b, b]$ is isomorphic to $[a \wedge b, a] \times[a \wedge b, b]$.

For modular lattices in general, the sublattice generated by $[a \wedge b, a]$ and $[a \wedge b, b]$ need not be $[a \wedge b, a \vee b]$. However in our situation this does occur.

CoROLlaRY $3.8([12]$, Theorem 3.3). $\mathscr{V}(\boldsymbol{O B G}) \cong \mathscr{C}(\boldsymbol{B}) \times \mathscr{V}(\boldsymbol{G})$.

Proof. Using, for example, the results of [7, Chapter VI], we have that any orthodox band of groups is a subdirect product of its maximum $\boldsymbol{B}$-image and its maximum $\boldsymbol{S} \boldsymbol{L} G$-image (since the inverse completely regular semigroups are just the semilattices of groups).

Thus if $S \in \boldsymbol{K}$ and $\boldsymbol{K} \subseteq \boldsymbol{O B} \boldsymbol{G}$, then $S \in(\boldsymbol{K} \cap \boldsymbol{B}) \vee(\boldsymbol{K} \cap \boldsymbol{S L} \boldsymbol{G})$. But by, for example, (6) of [13], 


$$
\begin{aligned}
\boldsymbol{K} \cap \boldsymbol{S L G} & =((\boldsymbol{K} \cap \boldsymbol{S} \boldsymbol{L G}) \cap \boldsymbol{S L}) \vee((\boldsymbol{K} \cap \boldsymbol{S} \boldsymbol{L} \boldsymbol{G}) \cap \boldsymbol{C S}) \\
& =(\boldsymbol{K} \cap \boldsymbol{S} \boldsymbol{L}) \vee(\boldsymbol{K} \cap \boldsymbol{G}),
\end{aligned}
$$

so $S \in(\boldsymbol{K} \cap \boldsymbol{B}) \vee(\boldsymbol{K} \cap \boldsymbol{G})$ (since $\boldsymbol{S} \boldsymbol{L} \leqq \boldsymbol{B}$ ).

Therefore $\boldsymbol{K}=(\boldsymbol{K} \cap \boldsymbol{B}) \vee(\boldsymbol{K} \cap \boldsymbol{S} \boldsymbol{L} \boldsymbol{G})$ and so $\boldsymbol{K}$ belongs to the sublattice generated by $\mathscr{V}(\boldsymbol{B})$ and $\mathscr{V}(\boldsymbol{G})$. Hence $\mathscr{V}(\boldsymbol{B})$ and $\mathscr{V}(\boldsymbol{G})$ generate $\mathscr{C}(\boldsymbol{B} \boldsymbol{G})$ and since $\boldsymbol{B} \cap \boldsymbol{G}=\boldsymbol{T}$ an application of Result 3.7 yields the result.

REMARK 3.9. Since the description of $\mathscr{V}(\boldsymbol{N B G})$ as a direct product of $\mathscr{Y}(\boldsymbol{S L})$ and $\mathscr{V}(\boldsymbol{C S})([13], \S 4)$ was essentially used in the proof of Theorem 3.1, we cannot of course apply the theorem to that situation.

4. A closure operator. For each subvariety $\boldsymbol{U}$ of $\boldsymbol{C R}$ we let $\boldsymbol{P}(\boldsymbol{U})$, or just $\boldsymbol{P} \boldsymbol{U}$, be the class consisting of those completely regular semigroups $S$ whose (completely regular) subsemigroups $e S e$ belong to $\boldsymbol{U}$ for every $e \in E_{S}$.

Proposition 4.1. For any variety $\boldsymbol{U} \cong \boldsymbol{C R}, \boldsymbol{P U}$ is a variety containing $\boldsymbol{U}$. In fact the operator $\boldsymbol{U} \rightarrow \boldsymbol{P} \boldsymbol{U}$ is a closure operator on $\mathscr{Y}(\boldsymbol{C R})$.

Proof. If $S \in \boldsymbol{P U}(\boldsymbol{U} \subseteq \boldsymbol{C R}), T$ is a (completely regular) subsemigroup of $S$ and $e \in E_{T}$ then $e \in E_{S}$ and $e T e$ is a (completely regular) subsemigroup of $e S e$ and so belongs to $U$. If $T$ is a morphic image of $S$, under $\phi$, say, and $e \in E_{T}$ then by a lemma of Lallement [8], $e=f \dot{\phi}$ for some $f \in E_{S}$, whence $e T e=(f S f) \phi \in \boldsymbol{U}$. That $\boldsymbol{P U}$ is closed under direct products is immediate upon noting that an element of a direct product of semigroups is idempotent if and only if each of its components is idempotent. Hence $\boldsymbol{P U}$ is a variety.

Clearly $\boldsymbol{U} \subseteq \boldsymbol{P} \boldsymbol{U}$, and $\boldsymbol{U} \subseteq \boldsymbol{V}$ implies $\boldsymbol{P} \boldsymbol{U} \subseteq \boldsymbol{P} \boldsymbol{V}$. If $S \in \boldsymbol{P}(\boldsymbol{P} \boldsymbol{U})$ and $e \in E_{S}$ then $e S e \in \boldsymbol{P U}$. But $e \in E_{e S e}$ and so $e S e \in \boldsymbol{U}$. Therefore $e S e \in \boldsymbol{U}$, whence $\boldsymbol{P}(\boldsymbol{P} \boldsymbol{U})=\boldsymbol{P} \boldsymbol{U}$.

We call $\boldsymbol{P} \boldsymbol{U}$ the variety of pseudo- $\boldsymbol{U}$ semigroups.

Lemma 4.2. $\boldsymbol{P}(\boldsymbol{B} \boldsymbol{G})=\boldsymbol{B} \boldsymbol{G}$.

Proof. Let $S \in P(B G), x, y \in S, x \mathscr{P} y$ and $s \in S$. Now $x \mathscr{R} y$ implies sx. Rsy. Let $e=x x^{-1}=y y^{-1}$. Then since $H_{e} \leqq e S e$, a band of groups, we have

$$
e s x=(e s e) x \mathscr{H}(e s e) y=e s y .
$$

But esx $\mathscr{J} s x \mathcal{J} s y \mathcal{L}$ esy so (since $\mathscr{f}$-classes of $S$ are completely simple) 


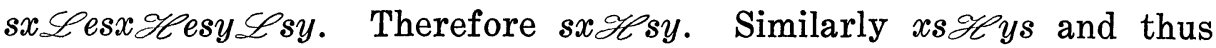
$\mathscr{H}$ is a congruence, that is $S \in \boldsymbol{B} G$.

As noted above $\boldsymbol{B} \boldsymbol{G} \subseteq \boldsymbol{P}(\boldsymbol{B} \boldsymbol{G})$ and the result follows.

As a result of this lemma, if $\boldsymbol{U} \subseteq B \boldsymbol{B}$, then $\boldsymbol{P} \boldsymbol{U} \subseteq \boldsymbol{B} \boldsymbol{G}$ also. Observe that $\boldsymbol{P}(\boldsymbol{T})=\boldsymbol{R B}$ and $\boldsymbol{P}(\boldsymbol{G})=\boldsymbol{C S}$. By Theorem 4.1 of [11], a completely regular semigroup $S$ is a normal band of groups if and only if it satisfies " $\mathscr{D}$-majorization": if $e, f, g \in E_{S}, e \geqq f, e \geqq g$, $f \mathscr{D} g$ then $f=g$. Clearly this is equivalent to saying each $\mathscr{D}$-class of $e S e$ contains precisely one idempotent for every $e \in E_{S}$, that is each $e S e \in \boldsymbol{S} \boldsymbol{L} \boldsymbol{G}$. Hence

LeMma 4.3. $\quad \boldsymbol{P}(\boldsymbol{S L G})=\boldsymbol{N B G}$ and $\boldsymbol{P}(\boldsymbol{S L})=\boldsymbol{N B}$.

If $\boldsymbol{U} \in \mathscr{Y}(\boldsymbol{C R})$, a congruence on a semigroup $S$ will be called a $\boldsymbol{U}$-congruence if $S / \rho \in \boldsymbol{U}$. If $S$ is completely regular then $S$ has a least $\boldsymbol{U}$-congruence. We now show how to derive the least $\boldsymbol{P} \boldsymbol{U}$-congruence on $S$ in terms of $\boldsymbol{U}$-congruences on the subsemigroups $e S e$, $e \in E_{S}$.

Proposition 4.4. If $\boldsymbol{U} \subseteq \boldsymbol{C R}$ and $\boldsymbol{S} \in \boldsymbol{C R}$ then the least $\boldsymbol{P} \boldsymbol{U}$-congruence on $S$ is the congruence generated by the union of the least $U$-congruences on the subsemigroups eSe, $e \in E_{S}$.

Proof. Denote by $\rho_{e}$ the least $\boldsymbol{U}$-congruence on $e S e, e \in E_{S}$, and put $\rho=\left(\cup\left\{\rho_{e}: e \in E_{S}\right\}\right)^{\sharp}, T=S / \rho$.

If $f \in E_{T}$ then, as above, $f=e \rho$ for some $e \in E_{S}$ and so $f T f \cong$ $(e S e) /(\rho \mid e S e)$. Since $\rho_{e} \subseteq \rho \mid e S e$, there is a morphism of $e S e / \rho_{e}$ upon $e S e /(\rho \mid e S e)$ and thus since $e S e / \rho_{e} \in \boldsymbol{U}, f T f \in \boldsymbol{U}$. Hence $T \in \boldsymbol{P U}$ and $\rho$ is a $\boldsymbol{P U}$-congruence.

Now let $\tau$ be an arbitrary $\boldsymbol{P U}$-congruence. For each $e \in E_{\boldsymbol{s}}$, $\tau \mid e S e$ is a congruence on $e S e$ and in fact a $\boldsymbol{U}$-congruence, since $e S e /(\tau \mid e S e) \cong(e \tau)(S / \tau)(e \tau) \in \boldsymbol{U}$ (since $e \tau \in E_{S / \tau}$ and $\left.S / \tau \in \boldsymbol{P} \boldsymbol{U}\right)$. Therefore $\rho_{e} \leqq \tau \mid e S e \leqq \tau$ for each $e \in E_{S}$ and $\rho \subseteq \tau$, by definition.

Hence $\rho$ is the least $\boldsymbol{P} \boldsymbol{U}$-congruence on $S$.

A result which is clearly relevant to this proposition and which is required in the next lemma is the following.

Proposition 4.5. If $S$ is any semigroup, $e \in E_{S}$ and $\rho$ is a congruence on $e S e$, then $\rho^{\sharp} \mid e S e=\rho$.

Proof. Clearly $\rho \subseteq \rho^{\sharp} \mid e S e$. Conversely, suppose $(x, y) \in \rho^{\sharp} \mid e S e$. Then there exists a sequence

$$
x=x_{0} \longrightarrow x_{1} \longrightarrow x_{2} \longrightarrow \cdots \longrightarrow x_{n}=y
$$


of elements of $S$ such that for each $i(1 \leqq i \leqq n), x_{i-1}=s_{i} a_{i} t_{i}$ and $x_{i}=s_{i} b_{i} t_{i}$ for some $s_{i}, t_{i} \in S^{1},\left(a_{i}, b_{i}\right) \in \rho$. Since $e x e=x$ and eye $=y$, the sequence may be replaced by the sequence

$$
x=e x_{0} e \longrightarrow e x_{1} e \longrightarrow e x_{2} e \longrightarrow \cdots \longrightarrow e x_{n} e=y,
$$

where for each $i, e x_{i-1} e=e s_{i} a_{i} t_{i} e=\left(e s_{i} e\right) a_{i}\left(e t_{i} e\right)$, since $a_{i} \in e S e$, and $e x_{i} e=\left(e s_{i} e\right) b_{i}\left(e t_{i} e\right)$ similarly. Since $\left(a_{i}, b_{i}\right) \in \rho$ and $e s_{i} e, e t_{i} e \in e S e$, $\left(e x_{i-1} e, e x_{i} e\right) \in \rho, 1 \leqq i \leqq n$, hence $(x, y) \in \rho$, as required.

The next lemma is required in $\S 5$.

Lemma 4.6. If $S \in \boldsymbol{C R}, e \in E_{S}$ and $\rho$ is a congruence on eSe such that $\rho \cap \mathscr{H}=\iota\left(\right.$ on eSe) then $\rho^{\sharp} \cap \mathscr{H}=\iota$ on $S$.

Proof. Suppose $(x, y) \in \rho^{\sharp} \cap \mathscr{H}, x, y \in S, x \neq y$. Then as above, $x=s_{1} a_{1} t_{1}$ for some $s_{1}, t_{1} \in S^{1}, a_{1} \in e S e$. Therefore $J_{x} \leqq J_{a_{1}} \leqq J_{e}$ and since $S$ is a semilattice of completely simple semigroups, exe $\mathscr{D} x$. Let $u \in R_{e x e} \cap L_{x}$ and let $u^{\prime}$ be the inverse of $u$ in $R_{x} \cap L_{e x e}$. Then $u x u^{\prime}, u y u^{\prime} \in H_{e x e}$ and we have (noting that $e S e$ consists of complete $\mathscr{H}$-classes of $S)\left(u x u^{\prime}, u y u^{\prime}\right) \in \rho^{\sharp} \mid e S e$. By the previous proposition, $\rho^{\#} \mid e S e=\rho$, so $u x u^{\prime}=u y u^{\prime}$, whence $x=u^{\prime}\left(u x u^{\prime}\right) u=u^{\prime}\left(u y u^{\prime}\right) u=y$, a contradiction.

5. Lattice joins. In this section we find the join $O \vee N B G$, and as a corollary $\boldsymbol{O B G} \vee \boldsymbol{N B G}$. From the results of [13], it may be observed that $\boldsymbol{O B G} \vee \boldsymbol{N B G}=\boldsymbol{B} \vee \boldsymbol{C S}$.

Since, as was seen in $\S 4, N B G=P(S L G)$ and $S L G \leqq O$ we have $N B G \subseteq P O$ and so $O \vee N B G \subseteq P O$. In particular $O B G \vee N B G \subseteq$ $\boldsymbol{P O B G}(=\boldsymbol{P O} \cap \boldsymbol{B} \boldsymbol{G})$. In view of the next example, $\boldsymbol{O B} \boldsymbol{B} \vee \boldsymbol{N B} \boldsymbol{G}$ is properly contained in $\boldsymbol{B} G$.

EXAMPLE 5.1. Let $C$ be a nonorthodox completely simple semigroup and $S=C^{1}$. Since $\mathscr{C}$ is a congruence on $C$, it is also a congruence on $S$, that is $S \in \boldsymbol{B G}$. But $S \notin P O B G$ since $S=1 . S .1$ is not orthodox.

Consider now the property:

$$
a p b \mathscr{H} a p p^{-1} b \text { for all } p \in\left\langle E_{S}\right\rangle, a, b \in S .
$$

Any band of groups satisfies $\left(^{*}\right)$ since of course $p \mathscr{H} p p^{-1}$ and $\mathscr{H}$ is a congruence on a band of groups. Moreover any orthodox completely regular semigroup also satisfies $\left(^{*}\right)$, for then $\left\langle E_{S}\right\rangle=E_{S}$ and $p=p p^{-1}$.

We now show $\left(^{*}\right)$ may be expressed in terms of identities and so the class $I$ of all completely regular semigroups satisfying $\left(^{*}\right)$ forms a subvariety of $\boldsymbol{C R}$ containing, as we have just seen $\boldsymbol{O} \vee \boldsymbol{B G}$.

For each $n \geqq 1$ let $I_{n}$ be the identity 


$$
\left(a p_{n} b\right)\left(a p_{n} b\right)^{-1}=\left(a p_{n} p_{n}^{-1} b\right)\left(a p_{n} p_{n}^{-1} b\right)^{-1}
$$

where $p_{n}=\left(x_{1} x_{1}^{-1}\right)\left(x_{2} x_{2}^{-1}\right) \cdots\left(x_{n} x_{n}^{-1}\right), \quad a, b, x_{i} \in S, i \geqq 1$. Since any product $p=e_{1} \cdots e_{n}$ of idempotents of $S$ can be expressed in the form $p=\left(e_{1} e_{1}^{-1}\right) \cdots\left(e_{n} e_{n}^{-1}\right)$ it is evident that a completely regular semigroup has property $\left({ }^{*}\right)$ if and only if it satisfies the set $\left\{I_{n}\right\}_{n \geqq 1}$ of identities. The main property of such semigroups which we exploit is the following.

Lemma 5.2. If $S \in I$ then the congruence on $S$ generated by $\mathscr{H}_{\left\langle E_{S}\right\rangle}$ is contained in $\mathscr{H}$. Hence the least O-congruence on $S$ is contained in $\mathscr{H}$.

Proof. Since $\mathscr{H}$ is an equivalence on $S$ it is sufficient to show that if $(p, q) \in \mathscr{H}_{\left\langle E_{S}\right\rangle}$ then $a p b \mathscr{H} a q b$ for all $a, b \in S^{1}$. But $p \mathscr{H} p p^{-1}=$ $q q^{-1} \mathscr{H} q$, so $a p b \mathscr{H} a p p^{-1} b=a q q^{-1} b \mathscr{H} a q b$, using (*).

The least $\boldsymbol{O}$-congruence is clearly generated by all pairs $\left((e f)^{2}, e f\right), e, f \in E_{S}$. Since $\left((e f)^{2}, e f\right) \in \mathscr{H}_{\left\langle E_{S}\right\rangle}$ the second statement follows immediately.

Our main theorem for this section is now

THEOREM 5.3. A completely regular semigroup $S \in \boldsymbol{O} \vee N B G$ if and only if $S$ is pseudo-orthodox and satisfies (*). That is, $\boldsymbol{O} \vee$ $N B G=P O I(=P O \cap I)$.

Proof. That any semigroup in $O \vee N B G$ is pseudo-orthodox and satisfies $\left(^{*}\right)$ has been established. The converse will be proved by showing that any such completely regular semigroup $S$ is a subdirect product of an orthodox semigroup and a normal band of groups; this will follow from our proof that, on such a semigroup, the least

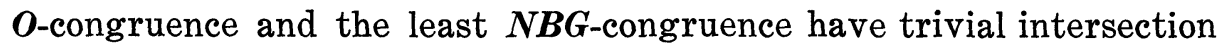
(see, for example [5], Theorem 20.2).

By the previous lemma the least $O$-congruence on $S, \alpha$, say, is contained in $\mathscr{H}$. Let $\eta$ be the least $N B G$-congruence on $S$. Since $\boldsymbol{N B} \boldsymbol{G}=\boldsymbol{P}(\boldsymbol{S L G}), \eta$ is the congruence generated by the union of the least $S L G$-congruences on the subsemigroups $e S e, e \in E_{S}$ (using Proposition 4.4). Since $\boldsymbol{S L G}$ is precisely the class of completely regular semigroups which are also inverse semigroups, the least $S L G$-congruence on $e S e$ is just the least inverse congruence $\mathscr{Y}_{\text {ese }}$. Moreover since $S \in P O$, each $e S e$ is orthodox and therefore $\mathscr{V}_{e S e} \cap$ $\mathscr{H}=\iota$ on $e S e$ (see, for example [7, p. 191]). By Lemma 4.6, $\mathscr{Y}_{\text {eSe }} \cap \cap \mathscr{H}=\iota$ on $S$.

At this stage we apply the comment made in Remark 3.3: if $\left\{\tau_{k}\right\}_{k \in K}$ is a collection of congruences contained in $\mathscr{D}$ on a completely 
regular semigroup $S$, and if $\tau_{k} \cap \mathscr{H}=\iota$ for all $k \in K$, then $\vee\left\{\tau_{k}: k \in K\right\} \cap \mathscr{H}=\iota$ (the join denoting the join in the lattice of congruences on $S)$.

Therefore $\eta \cap \mathscr{H}=\left(\vee \mathscr{Y}_{e S e}{ }^{\sharp}\right) \cap \mathscr{H}=\iota$ and so for any $S \in P O I$ we have $\eta \cap \alpha \subseteq \eta \cap \mathscr{C}=\iota$, as required.

Corollary 5.4. $B \vee C S=O B G \vee N B G=P O B G$.

Proof. As noted earlier, $\boldsymbol{B} \boldsymbol{G} \subseteq \boldsymbol{I}$.

CoROLlaRY 5.5 (to the proof).

(i) Any semigroup in POI is a subdirect product of an orthodox semigroup and a normal band of groups (in fact of the maximum orthodox morphic image and the maximum NBG morphic image).

(ii) Any pseudo-orthodox band of groups is a subdirect product of a band and a normal band of groups.

Proof. (i) is immediate. From the proof of the theorem we see that if $S \in P O B G$ then $S$ is a subdirect of an orthodox band of groups and a normal band of groups. However more strongly, $\eta \cap$

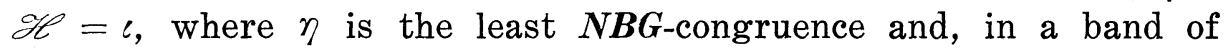
groups, $\mathscr{H}$ is the least B-congruence. Thus (ii) follows.

Before completing this section we show that pseudo-orthodoxy

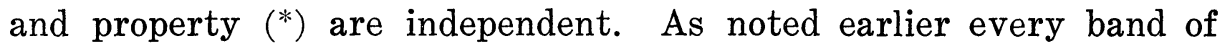
groups satisfies (*) but need not be pseudo-orthodox (Example 5.1). We now give an example to show that pseudo-orthodoxy need not imply $(*)$.

EXAMPLE 5.6. Let $C$ be a nonorthodox completely simple semigroup and let $c \rightarrow \bar{c}$ be a bijection of $C$ upon a disjoint set $R$. Define a product on $S=C \cup R$ by extending that on $C$, putting right zero product on $R$ and

$$
\begin{aligned}
& \bar{a} b=(\overline{a b}), \\
& a \bar{b}=\bar{b}, \quad \text { for all } a, b \in C .
\end{aligned}
$$

It is routine to verify that $S$ is a pseudo-orthodox completely regular semigroup.

Now let $e, f$ be two idempotents of $C$ whose product is nonidempotent and let $h$ be the idempotent in $H_{e f}$. Then

$$
\bar{e}(e f)=\overline{e f} \text { and } \bar{e} h=\overline{e h}=\bar{h}
$$

since $C$ is completely simple. Hence $S$ does not satisfy $\left({ }^{*}\right)$, for $(e f, h) \in \mathscr{H}_{\left\langle E_{S}\right\rangle}$ but $(\bar{e}(e f) \bar{e} h) \notin \mathscr{H}$ (since $R$ has trivial subgroups). 
Finally a direct decomposition similar to that of $\mathscr{V}(\boldsymbol{O B G})$ (Corollary 3.8) may be found for $[\boldsymbol{R B}, \boldsymbol{P O B G}]$, using Corollary 5.5.

Corollary 5.7. [RB, POBG] $\cong[R B, B] \times[R B, C S]$.

Proof. Since $\boldsymbol{B} \cap \boldsymbol{C S}=\boldsymbol{R B}$ it will be sufficient, by Result 3.7, to show that $[\boldsymbol{R B}, \boldsymbol{B}]$ and $[\boldsymbol{R B}, \boldsymbol{C S}]$ generate $[\boldsymbol{R B}, \boldsymbol{P O B G}]$.

Let $\boldsymbol{K} \cong \boldsymbol{P O B G}$ and $S \in \boldsymbol{K}$. By Corollary $3.8, S$ is a subdirect

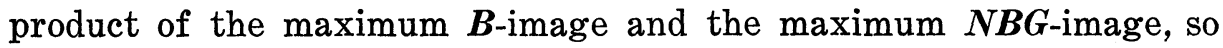
that $S \in(\boldsymbol{K} \cap \boldsymbol{B}) \vee(\boldsymbol{K} \cap \boldsymbol{N B G})$ and $\boldsymbol{K}=(\boldsymbol{K} \cap \boldsymbol{B}) \vee(\boldsymbol{K} \cap \boldsymbol{N B G})$.

Applying (6) of [13],

$$
\begin{aligned}
\boldsymbol{K} \cap \boldsymbol{N B G} & =((\boldsymbol{K} \cap \boldsymbol{N B G}) \cap \boldsymbol{S L}) \vee((\boldsymbol{K} \cap \boldsymbol{N B G}) \cap \boldsymbol{C S}) \\
& =(\boldsymbol{K} \cap \boldsymbol{S L}) \vee(\boldsymbol{K} \cap \boldsymbol{C S}) \\
\boldsymbol{K} & =(\boldsymbol{K} \cap \boldsymbol{B}) \vee(\boldsymbol{K} \cap \boldsymbol{C S})
\end{aligned}
$$

so

Thus if $\boldsymbol{K} \in[\boldsymbol{R B}, \boldsymbol{P O B G}], \boldsymbol{K}$ belongs to the lattice generated by $[R B, B]$ and $[R B, C S]$, as required.

REMARK 5.8. By generalizing the methods of [12] this corollary may be proved directly (with rather more difficulty).

Added in Proof. Since this paper was accepted, the authors have learned that our Corollary 5.4 has also been obtained by V. V. Rasin, "On varieties of bands of groups", in XV All-Union Algebra Conference, 1979.

\section{REFERENCES}

1. A.P. Birjukov, Varieties of idempotent semigroups, Algebra i Logika, 9 (1970), 255-273.

2. C. F. Fennemore, All varieties of bands, Math. Nachr., 48 (1971), I: 237-252, II: 253-262.

3. D. G. Fitzgerald, On inverses of products of idempotent in regular semigroups, J. Austral. Math. Soc., 13 (1972), 335-337.

4. J.A. Gerhardt, The lattice of equational classes of idempotent semigroups, J. Algebra, 15 (1970), 195-224.

5. G. Grätzer, Universal Algebra, Van Nostrand, Princeton, 1968.

6. - General Lattice Theory, Birkhauser Verlag, Basel, 1978.

7. J. M. Howie, An Introduction to Semigroup Theory, Academic Press, London, 1976.

8. G. Lallement, Congruences équivalences de Green sur un demi-groupe régulier, C.R. Acad. Sci. Paris, Sér. A, 262 (1966), 613-616.

9. W.D. Munn, A certain sublattice of the lattice of congruences on a regular semigroup, Proc. Cambridge Phil. Soc., 60 (1964), 385-391.

10. B. H. Neumann, Universal Algebra, Lecture Notes, Courant Inst. of Math. Sci., New York University.

11. M. Petrich, Regular semigroups satisfying certain conditions on idempotent and ideals, Trans. Amer. Math. Soc., 170 (1972), 245-267.

12. — Varieties of orthodox bands of groups, Pacific J. Math., 58 (1975), 209-217. 
13. M. Petrich, Certain varieties and quasivarieties of completely regular semigroups, Canad. J. Math., 29 (1977), 1171-1197.

14. C. Spitznagel, The lattice of congruences on a band of groups, Glasgow Math. J., 14 (1973), 189-197.

Received August 15, 1979 and in revised form February 15, 1980. The second author gratefully acknowledges the support of a Monash University Research Fellowship.

MONASH UNIVERSITY,

Clayton, Victoria, Australia, 3168 



\section{PACIFIC JOURNAL OF MATHEMATICS}

\section{EDITORS}

DONALD BABBITT (Managing Editor)

University of California

Los Angeles, CA 90024

Hugo RossI

University of Utah

Salt Lake City, UT 84112

C. C. MOORE and ANDREW OGG

University of California

Berkeley, CA 94720

\section{J. DugundjI}

Department of Mathematics

University of Southern California

Los Angeles, CA 90007

R. FinN and J. Milgram

Stanford University

Stanford, CA 94305

\section{ASSOCIATE EDITORS}
E. F. BeCKENBACH
B. H. Neumann
F. WoLF
K. YoSHIDA

\section{SUPPORTING INSTITUTIONS}

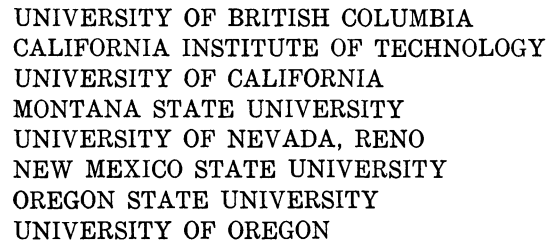

UNIVERSITY OF BRITISH COLUMBIA CALIFORNIA INSTITUTE OF TECHNOLOGY UNIVERSITY OF CALIFORNIA MONTANA STATE UNIVERSITY UNIVERSITY OF NEVADA, RENO NEW MEXICO STATE UNIVERSITY OREGON STATE UNIVERSITY UNIVERSITY OF OREGON

\author{
UNIVERSITY OF SOUTHERN CALIFORNIA \\ STANFORD UNIVERSITY \\ UNIVERSITY OF HAWAII \\ UNIVERSITY OF TOKYO \\ UNIVERSITY OF UTAH \\ WASHINGTON STATE UNIVERSITY \\ UNIVERSITY OF WASHINGTON
}

The Supporting Institutions listed above contribute to the cost of publication of this Journal, but they are not owners or publishers and have no responsibility for its content or policies.

Mathematical papers intended for publication in the Pacific Journal of Mathematics should be in typed form or offset-reproduced, (not dittoed), double spaced with large margins. Please do not use built up fractions in the text of the manuscript. However, you may use them in the displayed equations. Underline Greek letters in red, German in green, and script in blue. The first paragraph or two must be capable of being used separately as a synopsis of the entire paper. Please propose a heading for the odd numbered pages of less than 35 characters. Manuscripts, in triplicate, may be sent to any one of the editors. Please classify according to the scheme of Math. Reviews, Index to Vol. 39. Supply name and address of author to whom proofs should be sent. All other communications should be addressed to the managing editor, or Elaine Barth, University of California, Los Angeles, California, 90024.

50 reprints to each author are provided free for each article, only if page charges have been substantially paid. Additional copies may be obtained at cost in multiples of 50 .

The Pacific Journal of Mathematics is issued monthly as of January 1966. Regular subscription rate: $\$ 84.00$ a year (6 Vols., 12 issues). Special rate: $\$ 42.00$ a year to individual members of supporting institutions.

Subscriptions, orders for numbers issued in the last three calendar years, and changes of address shoud be sent to Pacific Journal of Mathematics, P.O. Box 969, Carmel Valley, CA 93924, U.S.A Old back numbers obtainable from Kraus Periodicals Co., Route 100, Millwood, NY 10546.

PUBLISHED BY PACIFIC JOURNAL OF MATHEMATICS, A NON-PROFIT CORPORATION

Printed at Kokusai Bunken Insatsusha (International Academic Printing Co., Ltd.). 8-8, 3-chome, Takadanobaba, Shinjuku-ku, Tokyo 160, Japan. 


\section{Pacific Journal of Mathematics}

\section{Vol. 91, No. 2 December, 1980}

Victor P. Camillo and Julius Martin Zelmanowitz, Dimension modules ... . . 249

Yonina S. Cooper, Stable sequences in pre-abelian categories ........... 263

Chandrakant Mahadeorao Deo and H. Ship-Fah Wong, On Berry-Esseen approximation and a functional LIL for a class of dependent random fields.........................................

H. P. Dikshit and S. N. Dubey, $|C, 1|$ summability of series associated with

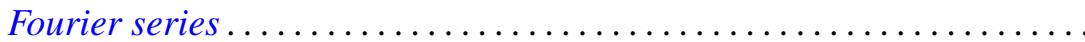

M. Edelstein, On the homomorphic and isomorphic embeddings of a semiflow into a radial flow.

Gilles Godefroy, Compacts de Rosenthal ..................... 293

James Guyker, Commuting hyponormal operators ................ 307

Thomas Eric Hall and Peter R. Jones, On the lattice of varieties of bands of

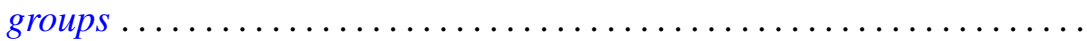

Taqdir Husain and Saleem H. Watson, Topological algebras with orthogonal Schauder bases ....................................

V. K. Jain, Some expansions involving basic hypergeometric functions of two variables. . .

Joe W. Jenkins, On group actions with nonzero fixed points ........... 363

Michael Ellsworth Mays, Groups of square-free order are scarce ........ 373

Michael John McAsey, Canonical models for invariant subspaces... 377

Peter A. McCoy, Singularities of solutions to linear second order elliptic partial differential equations with analytic coefficients by approximation methods...

Terrence Millar, Homogeneous models and decidability.

Stephen Carl Milne, A multiple series transformation of the very well poised

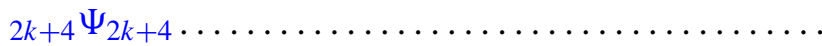

Robert Olin and James E. Thomson, Irreducible operators whose spectra are spectral sets...

Robert John Piacenza, Cohomology of diagrams and equivariant singular

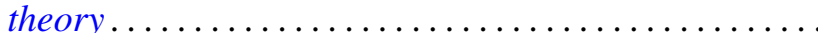

Louis Jackson Ratliff, Jr., Integrally closed ideals and asymptotic prime divisors

Robert Breckenridge Warfield, Jr., Cancellation of modules and groups and stable range of endomorphism rings.................

B. J. Day, Correction to: "Locale geometry" ...............

Stanley Stephen Page, Correction to: "Regular FPF rings" ... 